\title{
Considerations for evaluating the effectiveness and long- term outcome of enzyme replacement therapy in Pompe disease
}

\author{
Chong Kun Cheon, MD, PhD \\ Department of Pediatrics, Pusan National University Children's Hospital, Yangsan, Korea
}

Glycogen storage disease type II, also known as Pompe disease, is a rare, progressive disorder. It is characterized by partial or total deficiency of acid alpha-glucosidase (GAA), an enzyme that hydrolyzes lysosomal glycogen. ${ }^{1)}$ As a result of deficiency of GAA enzyme, lysosomal glycogen accumulates in tissues of the body, which can lead to progressive debilitation, organ failure, and death. The diagnosis of Pompe disease in children and adults can be challenging because of the heterogeneous clinical presentation and considerable overlap of signs and symptoms identified in other neuromuscular diseases. ${ }^{1)}$ The infantile-onset form of the disease is rapidly progressive which is characterized by marked cardiomyopathy, respiratory failure, and death within the first year of life. ${ }^{2)}$ Late-onset Pompe disease can present from the age of 1 year to the sixth decade of life which is characterized by progressive proximal skeletal muscle weakness and respiratory insufficiency that leads to respiratory failure and death. ${ }^{3,4)}$ Enzyme replacement therapy (ERT) using recombinant human acid alpha-glucosidase was developed as the most promising therapeutic approach for Pompe disease although the clinical response is variable; however, the evidence is lacking, especially regarding the optimal dose or dose frequency and the effect of long-term alglucosidase alfa treatment on extended survival as well as ventilation-free survival and cardiomyopathy. ${ }^{5)}$ Recently, Kim et al. ${ }^{6}$ published clinical features, genotypes, changes before and after ERT, and long-term outcomes in 5 Korean patients with infantile-onset Pompe disease (IOPD) and late-onset Pompe disease (LOPD). During a median 10-year ERT, there remains stable in the abnormalities shown on initial echocardiogram in patients with IOPD, along with improved organomegaly and normalization of liver transaminase level in patients with late-onset Pompe disease without adverse effects. Usually, clinical effectiveness in Pompe disease is evaluated by ventilator-free survival, reduction in left ventricular mass index, and improvement of motor function. The study by Kim et al. ${ }^{6}$ was well conducted on this score to assess clinical effectiveness including quality of life in patients with Pompe disease. To evaluate the functional exercise capacity, there are several modalities available. The modality used should be chosen based on the clinical questions to be addressed and on available resources. ${ }^{7)}$ The most popular clinical exercise tests in the order of increasing complexity are stair climbing, a 6-minute walk test (6-MWT), a shuttle-walk test, a cardiac stress test (e.g., Bruce protocol), and a cardiopulmonary exercise test. ${ }^{8)}$ A recent review of functional walking tests concluded that "the 6-MWT is easy to administer, better tolerated, and more reflective of activities of daily living than the other walk tests. ${ }^{9)}$ The 6-MWT is a practical and simple test that requires a $100-\mathrm{ft}$ hallway but no exercise equipment or advanced training for technicians are needed., ${ }^{6,7)}$ It assesses the global and integrated responses of all the systems involved during the exercise, including the cardiovascular and pulmonary systems, systemic and peripheral circulation, blood, neuromuscular units, and muscle metabolism. $\left.{ }^{9}\right)$ However, as a 6-MWT can be evaluated only in patients capable of independent walking, Kim et al. ${ }^{6}$ conducted a motor function measurement using manual muscle testing method to overcome these limitations in patients incapable of independent walking.

The efficacy of ERT in patients with Pompe disease is affected by cross-reactive immunological material (CRIM) status and the presence of symptoms. CRIM-negative status has been recognized as a poor prognostic factor in Pompe disease. ${ }^{10)}$ CRIMnegative patients make no GAA protein and develop highly sustained antibody titers to ERT that render the treatment ineffective. ${ }^{10)}$ Antibody titers are generally low for the majority of CRIM-positive patients and there is typically a better clinical outcome. ${ }^{10)}$ Therefore, knowledge of CRIM status is important before ERT is administered. However, CRIM testing is not available in Korea at present, and there is a difficult part of commissioning a sample for research within a short period of time to an overseas institution when performing a test. In a study conducted by Kim et al., ${ }^{6}$ they measured neutralizing antibodies affecting the bioavailability of recombinant enzyme and found all 3 patients with infantile-onset Pompe disease had no alphaglucosidase $\operatorname{IgG}$ antibody, 2 patients with late-onset Pompe disease showed positive alpha-glucosidase $\operatorname{Ig} G$ antibody level.

Corresponding author: Chong Kun Cheon, MD, PhD. Department of Pediatrics, Pusan National University Children's Hospital, Pusan National University School of Medicine, Geumo-ro 20, Yangsan 50612, Korea 
As this retrospective clinical study cannot directly evaluate mechanisms by which alpha-glucosidase IgG titers impact on the outcomes of ERT, more data are still needed from studies with prospective study design and a representative sample to get further insight of potential long-term outcomes in the Korean patients with Pompe disease.

See the article "Clinical and molecular characterization of Korean children with infantile and late-onset Pompe disease: 10 years of experience with enzyme replacement therapy at a single center" in Volume 62 on page 224.

\section{Conflicts of interest}

No potential conflict of interest relevant to this article was reported.

\section{References}

1. Kishnani PS, Howell RR. Pompe disease in infants and children. J Pediatr 2004;144(5 Suppl):S35-43.
2. Kishnani PS, Hwu WL, Mandel H, Nicolino M, Yong F, Corzo D, et al. A retrospective, multinational, multicenter study on the natural history of infantile-onset Pompe disease. J Pediatr 2006;148:671-6.

3. Hagemans ML, Winkel LP, Van Doorn PA, Hop WJ, Loonen MC, Reuser AJ, et al. Clinical manifestation and natural course of late-onset Pompe's disease in 54 Dutch patients. Brain 2005;128(Pt 3): 671-7.

4. Laforêt P, Nicolino M, Eymard PB, Puech JP, Caillaud C, Poenaru L, et al. Juvenile and adult-onset acid maltase deficiency in France: genotypephenotype correlation. Neurology 2000;55:1122-8.

5. Chen M, Zhang L, Quan S. Enzyme replacement therapy for infantileonset Pompe disease. Cochrane Database Syst Rev 2017;11: CD011539.

6. Kim MS, Song A, Im M, Huh J, Kang IS, Song J, et al. Clinical and molecular characterization of Korean children with infantile and lateonset Pompe disease: 10 years of experience with enzyme replacement therapy at a single center. Korean J Pediatr 2019;62:224-34.

7. ATS Committee on Proficiency Standards for Clinical Pulmonary Function Laboratories. ATS statement: guidelines for the six-minute walk test. Am J Respir Crit Care Med 2002;166:111-7.

8. Weisman IM,Zeballos RJ. An integrated approach to the interpretation of cardiopulmonary exercise testing. Clin Chest Med 1994;15: 421-45.

9. Solway S, Brooks D, Lacasse Y, Thomas S. A qualitative systematic overview of the measurement properties of functional walk tests used in the cardiorespiratory domain. Chest 2001;119:256-70.

10. Bali DS, Goldstein JL, Banugaria S, Dai J, Mackey J, Rehder C, et al. Predicting cross-reactive immunological material (CRIM) status in Pompe disease using GAA mutations: lessons learned from 10 years of clinical laboratory testing experience. Am J Med Genet C Semin Med Genet 2012;160C:40-9. 